\title{
Ubber das Fließen in kapillaren Räumen
}

\author{
Von \\ H. GREINACHER
}

Mit 7 Abbildungen im Text

(Eingegangen am 14. 8. 58)

\section{Zusammenfassung}

Zunächst wurden die Strömungsverhältnisse in einer zylindrischen Kapillare, als dem Prototyp eines porösen Kanals, untersucht. Es wurden die Bewegungsgleichungen für die Flüssigkeitsfront aufgestellt für den Fall, daß eine Kapillare in eine Flüssigkeit eingetaucht wird; zunächst bei horizontaler Lage (reine Kapillarwirkung), sodann bei vertikaler Lage (zusätzliche Schwerewirkung). In ähnlicher Weise ließ sich auch die Formel für das Kapillarmodell eines porösen Körpers bei radialer Anordnung aufstellen. Mit den gewonnenen Resultaten konnten sodann die Strömungsverhältnisse in zweidimensionalen Körpern (wie Papier und Tonplättchen) verglichen werden. Besonders untersucht wurden poröse Streifen mit einseitiger und Kreisscheiben mit zentraler Benetzung. Es ließ sich zeigen, daß, wie bei Kapillaren, die Fließfront mit $\sqrt{t}$ fortschreitet, hier allerdings erst bei größeren Wegstrecken. Bei radialer Strömung ergaben sich Abweichungen von diesem Verhalten. Auf diese sowohl als auf den Umstand, daß bei linearer Strömung am Anfang das $\sqrt{t}$-Gesetz nicht erfüllt ist, wurde noch besonders eingegangen. Es ergab sich ferner die Frage, inwieweit der Verlauf der Fließkurven bei vertikaler Lage auch quantitativ mit dem in Kapillaren übereinstimmt. Zu diesem Zweck wurde eine dynamische Methode zur Steighöhenbestimmung in Kapillaren aufgezeigt und ihre Anwendbarkeit auf poröse Stoffe geprüft. Schließlich interessierte noch die Frage nach der Strömung unter alleiniger Wirkung der Schwere, im einzelnen nach der Abhängigkeit der Fließgeschwindigkeit von der Fließlänge. Inwieweit überhaupt die Voraussetzung einer gerichteten Strömung erfüllt ist, lassen die beigefügten Stromlinienbilder erkennen.

Nach Duclaudx und ERRera ${ }^{1}$ läßt sich die Kapillare eines Viskosimeters durch einen porösen Körper ersetzen. Umgekehrt kann man, um das Fließen in einem porösen Körper zu beschreiben, modellmäßig das Bild eines Bündels feiner Haarröhrchen verwenden. Da die Gesetze für zylindrische Kapillaren bekannt und einfach sind, läßt sich der

1 J. Duclaux und J. Errera, J. Physique Radium 6 (1925) 202. 
Kapillardurchmesser 2r und damit die Porenweite unschwer bestimmen. Dies wurde bereits von BECHHOLD ${ }^{2}$ nach zwei Methoden ausgeführt. Die eine bestand in der Messung des Druckes, den es braucht, um eine Flüssigkeit unter Überwindung des Kapillardruckes $p=\frac{2 \alpha}{r}$ durch eine Kapillare hindurchzutreiben (statische Methode), die andere fußte auf der Anwendung des Poiseuilleschen Gesetzes.

$$
V / t=\frac{\pi}{8} \frac{r^{4} p}{\eta l}
$$

(dynamische Methode). Da die Porenweite des ausgemessenen Ultrafilters nicht einheitlich war, ergab die erste Methode wesentlich größere Werte als die zweite, entsprechend dem Umstand, daß die erste die größte Porenweite, die zweite aber nur einen gewissen Mittelwert anzeigt. Durch Kombination beider Verfahren gelang es ERBE ${ }^{3}$ die Größenverteilung der Poren zu bestimmen. Diese Methoden wurden dann weiter ausgebaut durch MaNegold, Komagata und Albrecht 4 , die sich auch eingehend mit den verschiedenen Kapillarsystemen befaßten und auf die beiden Grenzfälle der Querschnittsform hinwiesen: kreisförmig und schlitzförmig. Für den letzteren Fall ist Formel (1) zu ersetzen durch

$$
V / t=\frac{2}{3} \frac{d^{3} b p}{\eta l}
$$

$2 d=$ Weite, $b=$ Breite des Schlitzes. Nach WICkE ${ }^{5}$ sind je nach Struktur des porösen Körpers 3 verschiedene Porensysteme zu unterscheiden. Eines davon kann modellmäßig durch ein Bündel zylindrischer Kanäle dargestellt werden, während für die beiden anderen, das kombinierte Makro- und Mikrosystem, sowie für die Schaum- bzw. Schwammstruktur andere Modelle zu wählen sind. Immerhin kann die zylindrische Kapillare als Prototyp eines porösen Kanals gelten, und es ist dann im einzelnen Falle zu prüfen, inwieweit die für Kapillaren abgeleiteten Gesetze zutreffend sind.

Im folgenden wurde das Verhalten von 2 dimensionalen porösen Gebilden wie Lösch-, Filter- und Chromatographiepapier, sowie von Tonplättchen beim Durchsaugen von Flüssigkeit näher untersucht und

2 H. BeChноLD, Z. physik. Chem. 64 (1908) 328.

3 F. ERBE, Kolloid-Z. 63 (1933) 277.

4 E. Manegold, S. Komagata und E. Albrecht, Kolloid-Z. 93 (1940) 166.

5 E. WICKE, Z. Elektrochem., Ber. Bunsenges. physik. Chem. 60 (1956) 774. 
mit dem in Kapillaren verglichen. Vor allem interessierte das Fortschreiten der Flüssigkeitsfront im Prüfobjekt, wenn dieses an gegebener Stelle mit Flüssigkeit in Berührung gebracht wird. Es wurden folgende Anordnungen, wie sie z. T. in ähnlicher Weise auch in der Papierchromatographie vorliegen, verwendet: 1a) Papierstreifen in horizontaler Lage, am einen Ende benetzt, 1 b) Papierstreifen in vertikaler Lage, mit den beiden Varianten $1 b_{1}$ ) Benetzung oben, $1 b_{2}$ ) Benetzung unten, 2) kreisförmiges Papierblatt, in der Mitte benetzt.

\section{Fließen in Kapillaren}

Zunächst seien die Verhältnisse im Kapillarmodell für Anordnung 1) näher untersucht. Die 3 Fälle $1 a, 1 b_{1}$ und $1 b_{2}$ sind schematisch in Abb. 1 dargestellt.

Fall1a. Hier wirkt nur die kapillare Saugkraft $K$. Durch diese wird einerseits die im Röhrchen befindliche und andererseits die bei $A$ in die Kapillare eintretendeFlüssig. keit beschleunigt. Die erstere erfährt die Beschleunigung

$$
m \ddot{x}=\pi r^{2} \varrho x \ddot{x},
$$

die zweite, die durch $d m$ $=\pi r^{2} \varrho d x$ gegeben ist, wird in der Zeit $d t$ von $o$ auf $v$ beschleunigt. Die Beschleunigung ist somit

$$
v^{\pi r^{2} \varrho d x} \overline{d t}=\pi r^{2} \varrho \dot{x}^{2} .
$$

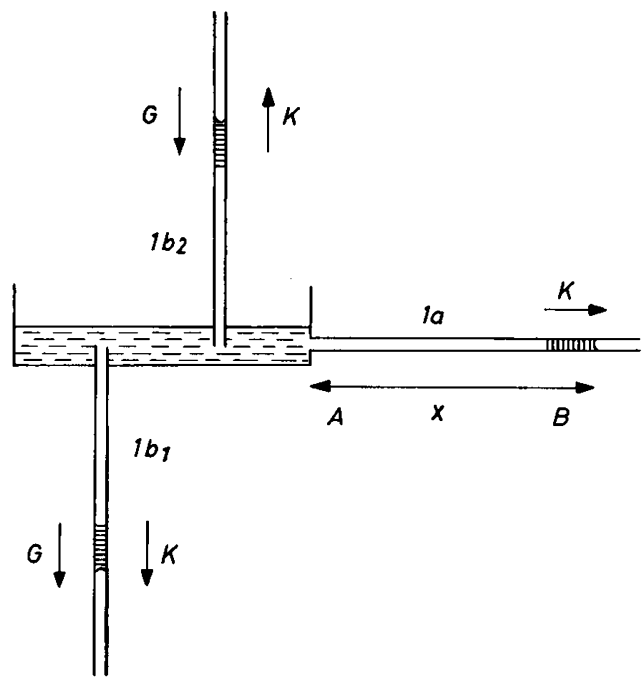

Abb. 1. Kapillar-Schema

Als weitere Kraft wirkt dem Kapillarzug noch die der inneren Reibung $K_{i}$ entgegen, so daß wir für die Bewegungsgleichung der Flüssigkeit den Ausdruck erhalten

$$
\pi r^{2} \varrho x \ddot{x}+\pi r^{2} \varrho \dot{x}^{2}=K-K_{i} .
$$

Für $K$ ist zu setzen $K=2 \pi r \alpha\left(\alpha=\right.$ Kapillarkonstante). $K_{i}$ finden wir aus dem Poiseuilleschen Gesetz (1), wenn wir bedenken, daß $K_{i}=\pi r^{2} p$ und die mittlere Strömungsgeschwindigkeit $v=V / t: \pi r^{2}$. Es ist danach $(l=x$ gesetzt)

$$
K_{i}=8 \pi \eta v x=8 \pi \eta \dot{x} x .
$$


Die Bewegungsgleichung lautet somit

$$
r^{2} \varrho\left(x \ddot{x}+\dot{x}^{2}\right)=2 r \alpha-8 \eta \dot{x} x .
$$

Das Strömungsgesetz erweist sich also als kompliziert, und einfache Verhältnisse sind nur zu erwarten, wenn die Glieder links vernachlässigt werden dürfen. Dies ist der Fall, wenn man nur den Strömungsverlauf nach längerer Zeit ins Auge faßt, d. h. wenn sowohl die Beschleunigung $\ddot{x}$ als die Geschwindigkeit $\dot{x}$ genügend klein geworden sind. Günstig wirkt sich dabei der Umstand aus, daß die Glieder links umgekehrtes Vorzeichen ergeben und sich so teilweise aufheben. Ferner ist zu beachten, daß links der Faktor $r^{2}$, rechts aber nur $r^{1}$ auftritt. Man wird daher im Falle genügend feiner Kapillaren (Poren) ohne großen Fehler die linke Seite von (5) gleich 0 setzen dürfen. Dies führt dann zu dem einfachen Resultat

$$
x^{2}=\frac{r \alpha}{2 \eta} \cdot t
$$

Fall $1 b$. Bei vertikaler Lage kommt als weitere Kraft noch das Gewicht der Flüssigkeitssäule, das $\pi r^{2} \varrho g x$ beträgt, hinzu. Die Bewegungsgleichung lautet daher jetzt

$$
2 \pi r \alpha \pm \pi r^{2} \varrho g x=8 \pi \eta \dot{x} x
$$

(- bei Aufwärts-, + bei Abwärtsbewegung). Die Integration ergibt

für $\uparrow$

$$
\begin{gathered}
e^{\lambda}(1-\lambda)=e^{-a t} \\
e^{-\lambda}(1+\lambda)=e^{-a t} .
\end{gathered}
$$

für $\downarrow$

Hier bedeutet $\lambda=\frac{x}{h}(h=$ Steighöhe $)$ und $a=\frac{r^{3} \varrho^{2} g}{16 \eta \alpha}$.

Da $\alpha=\frac{1}{2} h r \varrho g$, so ist auch

$$
a=\frac{r \alpha}{4 \eta h^{2}} .
$$

Für die Fließgeschwindigkeit $\lambda \cdot$ folgt

$$
\begin{array}{ll}
\text { für } \uparrow & \lambda \cdot=a\left(\frac{1}{\lambda}-1\right) 0<\lambda<1 \\
\text { für } \downarrow & \lambda \cdot=a\left(\frac{1}{\lambda}+1\right) 0<\lambda<\infty .
\end{array}
$$


Sie erreicht aufsteigend bei $\lambda=1, \mathrm{~d}$. h. bei $x=h$ den Wert 0 und absteigend bei $\lambda=\infty$ den Wert a. D. h. dann wird

oder

$$
\lambda \cdot=\frac{\dot{x}}{h}=\frac{v}{h}=\frac{r \alpha}{4 \eta h^{2}}
$$

$$
v=\frac{r \alpha}{4 \eta h}=\frac{r \alpha}{4 \eta} \cdot \frac{r \varrho g}{2 \alpha}=\frac{r^{2} \varrho g}{8 \eta} .
$$

Dies ist aber die Geschwindigkeit, mit der sich der Flüssigkeitsfaden in einer Kapillaren unter alleiniger Wirkung der Schwere nach unten bewegt. Es folgt dies unmittelbar auch aus (7), wenn man daselbst $\alpha=0$

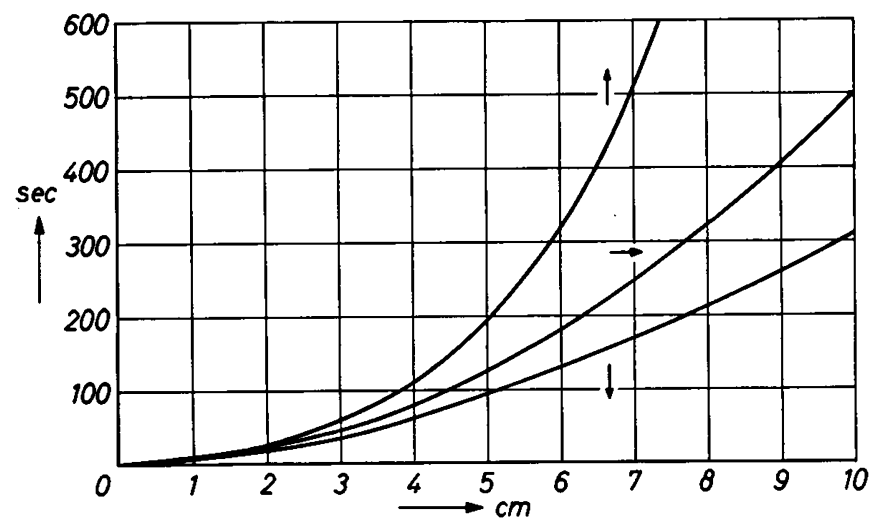

Abb. 2. Fließgeschwindigkeit in Kapillaren für die drei Richtungen $\uparrow \downarrow \rightarrow$

setzt. Der generelle Verlauf der 3 Kurven gemäß den Gl.(6), (8) und (9) ist unter Zugrundelegung der einfachen Zahlenwerte $h=a=1$ in Abb. 2 dargestellt.

In Wirklichkeit wäre für Wasser mit den Werten $\alpha_{18}=72,8$ dyn $\mathrm{cm}^{-1}$ und $\eta_{20}=0,0100 \mathrm{~g} \mathrm{~cm}^{-1} s^{-1}$ zu rechnen, d. h. mit den Werten $h=\frac{0,148}{r} \mathrm{~cm}$ und $a=8,26.10^{4} r^{3} s^{-1}$. Wenn man damit die Zeit ausrechnet, die Wasser zum Aufstieg auf z. B. 0,9 $h$ benötigt und diese vergleicht mit der Zeit $t^{\prime}$, die es für dieselbe Strecke bei horizontaler Lage gebrauchen würde, so findet man folgende Zahlen:

$\begin{array}{lcccc}r \mathrm{~mm} & h & 0,9 h & t & t^{\prime} \\ 1 & 1,48 \mathrm{~cm} & 1,33 \mathrm{~cm} & 0,017 s & 0,0049 s \\ 0,1 & 14,8 \mathrm{~cm} & 13,3 \mathrm{~cm} & 17 s & 4,88 \mathrm{~s} \\ 0,01 & 1,48 \mathrm{~m} & 1,33 \mathrm{~m} & 4,7 h & 1,35 h \\ 0,001 & 14,8 \mathrm{~m} & 13,3 \mathrm{~m} & 196 d & 56,4 d\end{array}$

$t / t^{\prime}$ besitzt in diesem Beispiel den Wert 3,47. 
Fall 2. Um den Fall radialer Strömung durch einen porösen Körper modellmäßig zu erfassen, denken wir uns diesen durch dicht gepackte radial angebrachte Kapillaren ersetzt. Im Abstand $x$ (Abb. 3) vom Zentrum seien auf dem Kranze der Dicke $d x n_{x}$ Röhrchen angebracht. Ist $v_{x}$ die Fließgeschwindigkeit an dieser Stelle, so ist die Reibungskraft

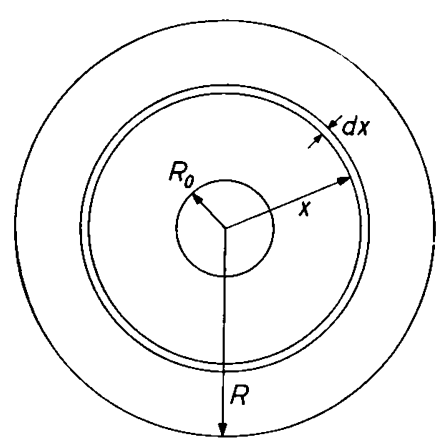

Abb. 3. Radiale Anordnung in einem Röhrchen $8 \pi \eta v_{x} d x$ und in allen Röhrchen zusammen

$$
d K_{i}=8 \pi \eta v_{x} n_{x} d x .
$$

Bedeutet $n_{R}$ die Zahl der Röhrchen an der Fließront $(R)$ und $v_{R}$ die Fließgeschwindigkeit daselbst, so lassen sich offenbar folgende Beziehungen anschreiben

$$
n_{x}=n_{R} \frac{x}{R} \text { und } v_{x}=v_{R} \frac{R}{x},
$$

so daß wir haben

$$
d K_{i}=8 \pi \eta v_{R} n_{R} d x
$$

und für die Reibungskraft in sämtlichen Röhrchen bis zur Fließfront

$$
K_{i}=8 \pi \eta v_{R} n_{R} \int_{R_{0}}^{R} d x=8 \pi \eta v_{R} n_{R}\left(R-R_{0}\right) .
$$

Dies ist nun gleich dem Kapillarzug $K$ an der Fließfront, für den wir schreiben können $K=2 \pi \delta K_{1}$, wo $\delta$ die Dicke der Schicht und $K_{1}$ den Kapillarzug pro $1 \mathrm{~cm}^{2}$ bedeutet. Drücken wir $n_{R}$ durch $n_{1} \mathrm{~d}$. h. die Zahl der Röhrchen pro $1 \mathrm{~cm}^{2}$ aus und setzen somit $n_{R}=2 \pi R \delta n_{1}$, so führt Gleichsetzen von $K$ und $K_{i}$ zu der Beziehung

$$
8 \pi \eta n_{1} v_{R}\left(R-R_{0}\right)=K_{1} .
$$

Da dieFließgeschwindigkeit an derFront $v_{R}=\frac{d R}{d t}$, so ergibt sich aus (15)

oder

$$
8 \pi \eta \int_{R_{0}}^{R} d R\left(R-R_{0}\right)=K_{1} / n_{1} \cdot t
$$

$$
4 \pi \eta\left(R-R_{0}\right)^{2}=K_{1} / n_{1} \cdot t .
$$

Da $K_{1} / n_{1}$ d. h. der Kapillarzug pro Röhrchen gleich $2 \pi r \alpha$ ist, so findet man schließlich

$$
\left(R-R_{0}\right)^{2}=\frac{r \alpha}{2 \eta} t
$$


Es ergibt sich also für die Fließstrecke $\left(R-R_{0}\right)$ bei radialer Strömung genau derselbe Ausdruck wie für die Fließstrecke $x$ bei einseitiger Strömung.

Die hier abgeleiteten Resultate sind unter der Annahme eines kreisförmigen Querschnittes der Kapillaren gewonnen. Es ist indessen nicht anzunehmen, daß sie für andere Querschnitte wesentlich anders ausfallen. Legt man z. B. den anderen Grenzfall eines schlitzförmigen Querschnittes zugrunde, so ergibt sich unter Benützung von (2) für das Fließen in horizontaler Richtung (Schlitzbreite groß gegen Weite vorausgesetzt)

$$
x^{2}=\frac{2}{3} \frac{\alpha d}{\eta} t .
$$

Dies entspricht aber ganz dem Ausdruck (6). Nur ist der Zahlenfaktor $\frac{1}{2}$ durch $\frac{2}{3}$ ersetzt. Die Porengröße $2 r$ bezw. $2 d$ ergibt sich demnach nahezu gleich groß. Die Rechnung ist daher für die anderen Fälle $1 \mathrm{~b}$ und 2 nicht weiter durchgeführt. Dagegen sei ergänzend noch der Ausdruck für das Poiseuillesche Gesetz bei radialer Strömung zwischen zwei parallelen Ebenen angegeben. Zwei Kreisscheiben, die in der Mitte ausgeschnitten sind (Radius: $R_{0}$ ), seien koaxial übereinander im Abstand $2 d$ angebracht. Die von $R_{0}$ bis $R$ strömende Flüssigkeit stehe unter dem Druck $p$. Es ergibt sich hierfür

$$
V / t=\frac{4 \pi d^{3} p}{3 \eta \ln R / R_{0}} .
$$

Für den Druck im Abstand $x$ vom Zentrum erhält man

$$
p_{x}=p \frac{\ln R / x}{\ln R / R_{0}} .
$$

Ausdruck (19) geht für $R$ und $R_{0}$ sehr groß, wenn $\operatorname{man} R-R_{0}=l$ setzt und $V / t$ pro Schlitzbreite $b=1 \mathrm{~cm}$ ausrechnet, wie es sein muß, in Formel (2) über.

\section{Fließen in porösen Materialien}

Fließen unter alleiniger Wirkung der Kapillarkraft

A. Eindimensionale Strömung. Vor allem interessierte die Frage, inwieweit auch für poröse Stoffe das quadratische Gesetz (4) Gültigkeit besitzt. Für die Versuche mit Wasser wurde die einfache Anordnung der Abb. 4 verwendet. Der mit einer Teilung versehene Papierstreifen $S$ von $2,4 \mathrm{~cm}$ Breite wurde in eine transparente Plastikhülle $H$ eingeschoben, so daß er diese nur mit den Seitenrändern berührte. Im 
Fond der Hülle befand sich ein mit Wasser getränkter Wattebausch $W$, um so eine Verdunstung des eingesaugten Wassers nach Möglichkeit zu verhindern. Die rechteckige Schale $G$ wurde bis oben mit Wasser gefüllt. Zu einem gegebenen Zeitpunkt legte man nun die Hülle mit dem

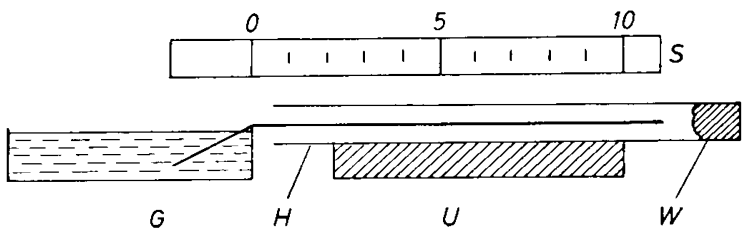

Abb. 4. Versuchsanordnung

Streifen bei der Marke Null auf den Rand der Wasserschale, wobei man durch eine passende Unterlage $U$ für horizontale Lage des Streifens sorgte. Bei genügend hoher Luftfeuchtigkeit konnte je nach Umständen die Plastikhülle auch weggelassen und der Streifen (Breite zumeist $3 \mathrm{~cm}$ )

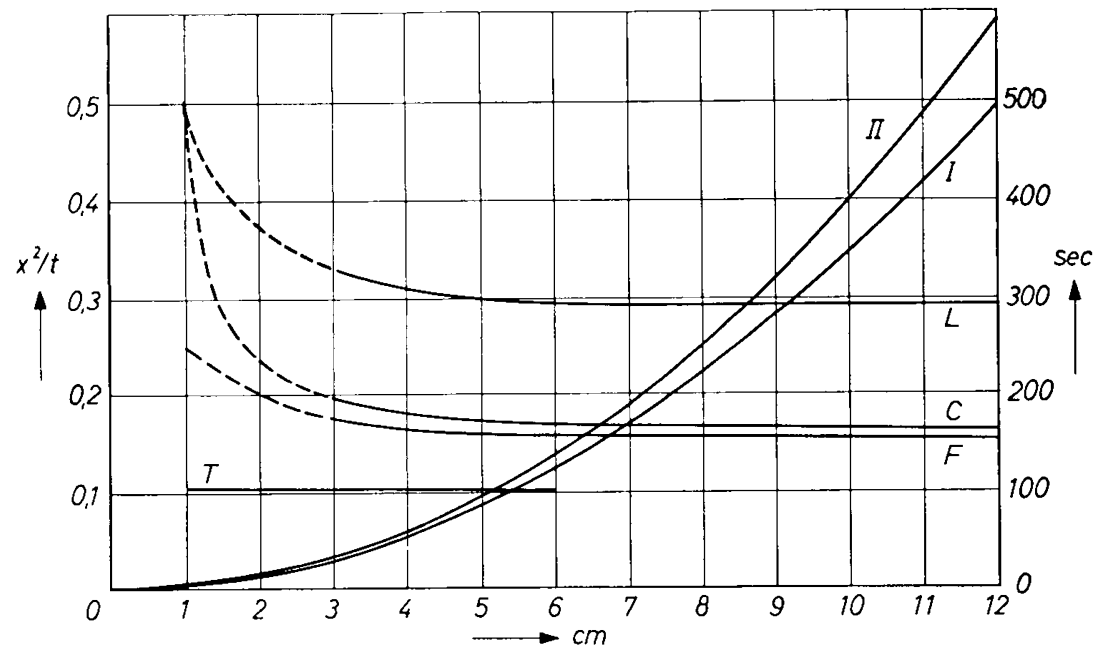

Abb. 5. 1. Fließgeschwindigkeit. I bei Verhinderung der Verdunstung; II ohne Verhinderung der Verdunstung. 2. Prüfung der Beziehung $x^{2} / t=$ const. für

$L$ Lösch-, $C$ Chromatographie- und $F$ Filtrierpapier, $T$ Tonplättchen

direkt auf die nicht benetzende Unterlage $U$ gelegt werden. Ein gewisses Interesse bot die Untersuchung von Tonplättchen $10 \times 10 \mathrm{~cm}^{2}$, Dicke: $5 \mathrm{~mm}$, da dieses Material die Voraussetzung zur Herstellung der neueren Diffusionshygrometer ${ }^{6}$ bildet. Hier erfolgte die Benetzung

${ }^{6}$ H. Greinacher, Helv. physica Acta 17 (1944) 437. Chimia [Zürich] 7 (1953) 11. Schweiz. Arch. angew. Wiss. Techn. 20 (1954) 198. 
der einen Seite des quadratischen Objekts dadurch, daß man sie in guten Kontakt mit nassem Löschpapier brachte. In allen Fällen ließ sich die Gleichmäßigkeit des Versuchsmaterials daran erkennen, daß die Fließfront sich geradlinig senkrecht zum Fluß fortbewegte.

In Abb.5 sind nun einige Messungen zusammengestellt. I und II zeigen den zeitlichen Verlauf für Löschpapier mit und ohne Plastikhülle bei einer Luftfeuchtigkeit von $30-40 \%$. II läßt eine deutliche Verlangsamung bei größeren Fließstrecken erkennen. Weiter ist zur Prüfung der Gültigkeit des quadratischen Gesetzes $x^{2} / t$ als Funktion von $x$ aufgetragen. Man erkennt, daß für alle 3 Papierproben $(C, F, L)$ bei nicht zu kleinen Wegstrecken (etwa von $5 \mathrm{~cm}$ an) annähernd horizontale Gerade herauskommen. Beim Tonplättchen $(T)$, für das $x^{2} / t$ im zehnfachen Maßstab aufgetragen wurde, ist das quadratische Gesetz sogar über den ganzen Bereich gut erfüllt. Die Ordinaten der Horizontalen entsprechen also dem Ausdruck der Formel (6). Rechnet man damit die entsprechenden Kapillarweiten $2 r$ aus, so erhält man folgende Werte:

$$
\begin{aligned}
& \text { F Filtrierpapier } 2 r=0,15 \cdot \frac{4 \eta}{\alpha}=8,2 \cdot 10^{-5} \mathrm{~cm} \\
& \text { C Whatman } 4 \quad 0,16 \cdot \frac{4 \eta}{\alpha}=8,8 \cdot 10^{-5} \mathrm{~cm} \\
& L\left\{\begin{array}{l}
\text { Löschblatt } \\
\text { Sihl Blotting }
\end{array} \quad 0,29 \cdot \frac{4 \eta}{\alpha}=15,9 \cdot 10^{-5} \mathrm{~cm}\right. \\
& T \quad \text { Tonplättchen } \quad 0,010 \cdot \frac{4 \eta}{\alpha}=0,55 \cdot 10^{-5} \mathrm{~cm} \text {. }
\end{aligned}
$$

Ergänzend sei bemerkt, daß auch Fließversuche mit Terpentinöl und mineralischem Nähmaschinenöl konstante Werte für $x^{2} / t$ ergaben. Infolge des langsameren Fließens fielen sie allerdings kleiner aus $(0,004$ bis 0,006$)$.

Charakteristisch an den $x^{2} / t$-Kurven ist ihr Ansteigen bei kleinen Wegstrecken. Das Einsaugen der Flüssigkeit erfolgt auf den ersten Zentimeter relativ rascher als später, $t$ ist kleiner als dem quadratischen Gesetz entspricht. Damit parallel geht die Tatsache, daß sich die Streifen auf den ersten $\mathrm{cm}$ wegs mit einer zusammenhängenden und damit regelmäßig spiegelnden Wasserschicht bedecken, während der übrige Teil eine matt scheinende Oberfläche zeigt und offenbar nicht maximal vollgesaugt ist.

Damit stehen folgende Erscheinungen in unmittelbarem Zusammenhang. Schneidet man einen Papierstreifen während des Einsaugens 
von der Wasserzufuhr ab, indem man ihn etwa durchschneidet, so wandert die Fließfront trotzdem noch eine Strecke weiter. Ferner beobachtet man, daß der Fließvorgang nicht aufhört, wenn die Fließfront am Ende des Streifens angelangt ist. Es ist dies an der spiegelnden Wasserfläche, die allmählich die ganze Länge überzieht, zu erkennen. Das Nachfließen läßt sich aber auch direkt sichtbar machen, indem man das Papier nach dem Stop der Fließfront mit Tusche betupft. Es entstehen dann je nach dem Abstand der Tupfe vom Papierende mehr oder weniger lange Tuschestriche $(\mathrm{Abb} .7 \mathrm{H})$. Es geht daraus hervor, daß auch hinter der Fließfront noch Kapillarkräfte wirksam sind und daß demnach das Ersatzmodell eines Bündels von Kapillaren, das nur an der Fließfront Kapillarität voraussetzt, die Verhältnisse zum mindesten für dünne Prüfobjekte nicht vollständig wiedergeben kann. Die Gültigkeit des quadratischen Gesetzes, sowie der Umstand, daß für $2 r$ Werte der richtigen Größenordnung gefunden werden, deuten aber darauf hin, daß bei größeren Fließstrecken während des Fließvorganges doch mit einem konstanten Querschnitt gerechnet werden kann. Auf kurzen Strecken tritt allerdings der Einfluß der Wasserhaut hervor. Nicht nur, daß der Querschnitt vergrößert ist, die Bewegung der Fließfront wird zudem durch das Fließen an der Oberfläche beschleunigt.

B. Radiale Strömung. Die der Abb. 3 entsprechende Anordnung wurde so realisiert, daß man in der Mitte eines kreisförmigen Löschblattes einen Kreis von $R_{0}=1 \mathrm{~cm}$ aussparte und die Wasserzufuhr durch einen dort hineingesteckten Wattepfropf besorgte, der seinerseits in ein Glas Wasser eintauchte. Ähnlich wurde mit einem Tonplättchen verfahren $\left(R_{0}=0,5 \mathrm{~cm}\right)$. Das Resultat war in Kürze folgendes: Für $\frac{\left(R-R_{0}\right)^{2}}{t}$ ergaben sich ähnliche Kurven wie für $x^{2} / t$ bei einseitiger Strömung. Die Werte waren aber sowohl für Löschpapier wie für das Tonplättchen nur etwa halb so groß. Die Strömung erfolgte also wesentlich langsamer als in Papierstreifen. Ferner blieb die Kurve auch bei größeren Wegstrecken leicht geneigt, so daß das quadratische Gesetz nur unvollkommen erfüllt war. Die Verhältnisse können also nicht durch das oben besprochene radiale Kapillarmodell wiedergegeben werden. Die geringere Fließgeschwindigkeit bei radialer Anordnung dürfte mit dem schon erwähnten Vorhandensein von Kapillarkräften hinter der Fließfront zusammenhängen bzw. mit dem Umstand, daß die hinter der Fließfront nachzufüllende Fläche bei radialer Anordnung mit dem Quadrat, bei einseitiger aber nur proportional zunimmt. Die 
radiale Anordnung läßt im übrigen unmittelbar die Fließgeschwindigkeit in allen Richtungen überblicken. Die Verschiedenheit, wie sie bei Chromatographiepapier ja bekannt ist, zeigte sich auch bei Löschblatt. Die elliptische Front ergab ein Achsenverhältnis von etwa 9:10.

\section{Fließen unter Wirkung von Kapillar- und Schwerkraft}

In Abb. 6 sind drei Kurven wiedergegeben, wie sie für die Fließgeschwindigkeit in aufsteigender $(\uparrow)$ und in absteigender $(\downarrow)$ im Vergleich zu der in horizontaler Richtung $(\rightarrow)$ gefunden wurden. Die Versuche sind mit Löschpapier von $3 \mathrm{~cm}$ Breite und $0,6 \mathrm{~mm}$ Dicke ausgeführt. Bei Verwendung dieses dickeren Materials und bei der relativ hohen Luftfeuchtigkeit von ca. $60 \%$ durfte der Verdunstungseffekt während des Einsaugens der Flüssigkeit als belanglos angenommen werden. Um vergleichbare Kurven zu erhalten, wurden die $\mathrm{Pa}$ pierstreifen nebeneinander aus demselben Material herausgeschnitten. Die Reproduzierbarkeit der Verhältnisse wurde durch Wiederholung des Fließversuchs in horizontaler Richtung noch besonders geprüft. Für eine

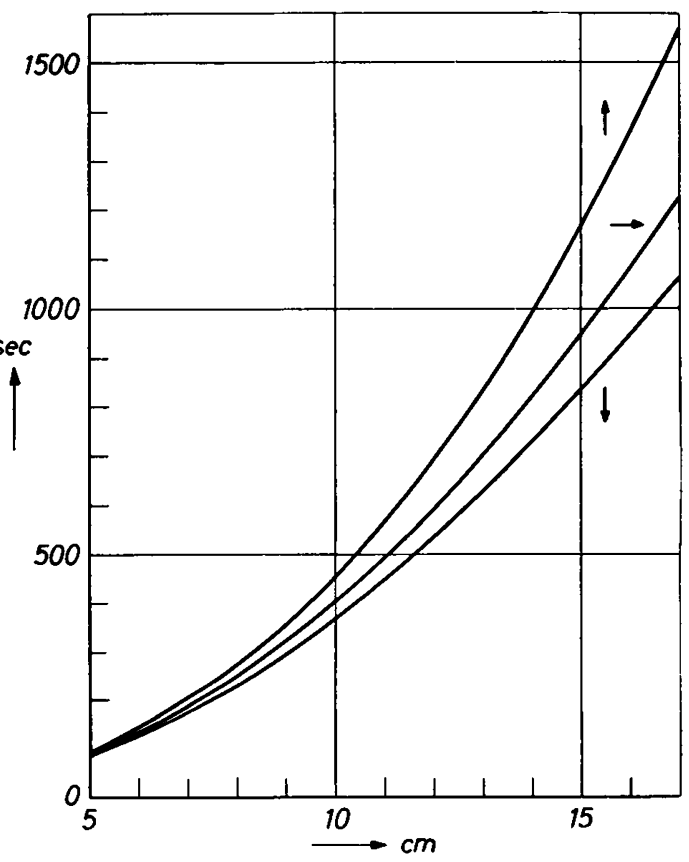

Abb.6. Fließgeschwindigkeit in Löschpapier für die drei Richtungen $\uparrow \downarrow \rightarrow$ Fließ-Strecke von $10 \mathrm{~cm}$ ergab sich beim einen Streifen $404 \mathrm{~s}$, beim anderen $423 s$. Der Versuch für das Aufsteigen der Flüssigkeit war leicht zu realisieren, indem man das untere Ende des Streifens zu einem gegebenen Zeitpunkt eintauchte. Bei dem für Abwärtsbewegung brachte man das Papier dadurch in Kontakt mit Wasser, daß man das eine umgebogene Ende des Streifens über den Rand eines Becherglases legte. Dieses war zur Vermeidung unerwünschter Kapillarwirkung 
paraffiniert und nur bis ca. $1 / 2 \mathrm{~cm}$ vom Rand mit Wasser gefüllt. Als Nullpunkt für die nach unten führende Fließ-Strecke wurde der höchste Punkt des Streifens gewählt. Dem unerwünschten Umstand, daß das Wasser bis zu diesem Punkte erst einige $\mathrm{mm}$ aufsteigen mußte, wurde dadurch nach Möglichkeit begegnet, daß man noch einen dicken ins Wasser eintauchenden Löschpapierstreifen auf den Kulminationspunkt auflegte.

Wie ersichtlich fallen die 3 Kurven bis etwa $5 \mathrm{~cm}$ wegs praktisch zusammen. Sie sind daher auch erst von dort an eingezeichnet. Im übrigen ergibt sich dasselbe typische Kurvenbild wie bei Kapillaren (Abb.2). Es kann indessen nicht ohne weiteres ersehen werden, ob die Resultate auch quantitativ übereinstimmen. Die Prüfung dieser Frage läßt sich folgendermaßen durchführen. Aus (11) und (12) gewinnt man eine einfache Beziehung, wenn man die Fließgeschwindigkeit $\dot{\lambda}$ im selben Abstand $x$ einmal bei Abwärts- und einmal bei Aufwärtsbewegung betrachtet. Nennen wir diese $\lambda_{1}$ und $\lambda_{2}$, so haben wir

$$
\begin{array}{cl} 
& \dot{\lambda}_{1}-\dot{\lambda}_{2}=2 a \\
\text { oder da } & \dot{\lambda}_{1}=\frac{\dot{x}_{1}}{h} \text { und } \dot{\lambda}_{2}=\frac{\dot{x}_{2}}{h} \\
& \dot{x}_{1}-\dot{x}_{2}=2 a h .
\end{array}
$$

Aus (10) und (6) folgt ferner

so daß wir erhalten

$$
a=\frac{x^{2}}{t} \cdot \frac{1}{2 h^{2}},
$$

$$
\dot{x}_{1}-\dot{x}_{2}=\frac{x^{2}}{t} \cdot \frac{1}{h} \text {. }
$$

Bezeichnen wir die konstante Größe $x^{2} / t$ mit $k$, so erhalten wir für die Steighöhe den Ausdruck

$$
h=\frac{k}{\dot{x}_{1}-\dot{x}_{2}} .
$$

Danach kann also $h$ aus der Fließgeschwindigkeit in den 3 Richtungen $\uparrow \downarrow \rightarrow$, d.h. nach einer dynamischen Methode, gefunden werden. Auf unseren Fall angewendet, wäre zu prüfen 1. ob gemäß (21) $\dot{x}_{1}-\dot{x}_{2}$ einen konstanten Wert aufweist, 2. ob, wenn ja, sich für $h$ ein brauchbarer Wert ergibt. Die erste Bedingung ist nun offenbar erfüllt, wenn man größere Fließ-Strecken ins Auge faßt. Da die Kurven dort praktisch geradlinig verlaufen, ist sowohl $\dot{x}_{1}$ als $\dot{x}_{2}$ und damit auch 
$\dot{x}_{1}-\dot{x}_{2}$ konstant. Hierfür ergibt sich ungefähr $0,0037 \mathrm{~cm} / \mathrm{s}$. Da man aus der Kurve $\rightarrow$ für $x^{2} / t$ den Wert 0,24 findet, so berechnet sich für die Steighöhe $65 \mathrm{~cm}$. Direkte Beobachtung ergab andererseits, da $\beta$ Wasser in 1 Stunde $23 \mathrm{~cm}$, in 2 Stunden $30 \mathrm{~cm}$ hoch stieg. Der nach (21) gefundene Wert dürfte daher vom richtigen nicht allzu weit entfernt sein. Für den zuzuordnenden Kapillarradius ergibt sich allerdings der relativ hohe Wert von $2,3 \cdot 10^{-3} \mathrm{~cm}$. Die Resultate zeigen somit, daß das Kapillarmodell die Verhältnisse nur teilweise quantitativ zu erfassen vermag.

\section{Fließen unter alleiniger Wirkung der Schwerkraft}

Hängt man einen Löschpapierstreifen an einem Ende über den Rand eines mit Wasser gefüllten Becherglases, so beobachtet man zunächst das Wandern der Fließfront unter Wirkung von Kapillar- und Schwerkraft zusammen. Ist die Front am unteren Ende angelangt, so fließt weiterhin Wasser unter reiner Schwerewirkung nach, und es findet Tropfenbildung statt. Denken wir uns das Prüfobjekt durch ein Bündel von Kapillaren ersetzt, in denen Poiseuillesche Strömung stattfindet, so können wir gemäß Formel (13) den entsprechenden Kapillarradius bestimmen. Es ist für die $n$ Röhrchen

$$
V / t=\frac{\pi r^{4} p}{8 \eta l} \cdot n
$$

Da das Druckgefälle $p / l$ im Schwerefeld $\varrho g$ beträgt und der Querschnitt $q=n \pi r^{2}$, so folgt

und damit

$$
V / t=\frac{r^{2} \varrho g q}{8 \eta}
$$

$$
r^{2}=\frac{8 \eta \cdot V / t}{\varrho g q}
$$

Indem man nach BechHoLd (1. c.) $q$ aus dem Leerraum des porösen Materials ableitet, läßt sich $r$ berechnen. Man kann aber schon, wenn man $q$ durch den größeren Querschnitt $Q$ des Objekts ersetzt, wenigstens eine untere Grenze für $r$ angeben und schreiben

$$
r>\sqrt{\frac{8 \eta \cdot V / t}{\varrho g Q}}
$$

So wurde für ein Löschpapier (Länge $18,5 \mathrm{~cm}$, Breite $3 \mathrm{~cm}$, Dicke $0,02 \mathrm{~cm}$ ) gefunden, daß in 162 Minuten $4,3 \mathrm{~g}$ Wasser abtropfte. Daraus 
ergibt sich $r>7,8 \cdot 10^{-4} \mathrm{~cm}$. Vergleichsweise wurde am selben Streifen auch der horizontale Fließversuch durchgeführt. Aus dem hiernach gefundenen Wert $x^{2} / t=0,3$ ergab sich indessen $r=0,8 \cdot 10^{-4} \mathrm{~cm}$. Dieser Wert entspricht etwa den Angaben, die Bechноцd über die Porengröße von Filterpapier macht. Der nach der Durchströmungsmethode gefundene Wert liegt somit um eine Größenordnung höher. Eine Erklärung hierfür liefert der Umstand, daß der Papierstreifen während des Abtropfens mit einer mehr oder weniger dicken Wasserschicht überzogen ist, erkennbar an der spiegelnden Oberfläche. Wenn nun ein Teil des Wassers über diese herabfließt, so ist dies gleichbedeutend mit einer Vergrößerung der mittleren Rohrweite. Wie dick die Wasserhaut ist, hängt weitgehend von der Anordnung ab. Sowohl die Höhe des Wasserniveau im Glas als die Partie des Streifens, die am Rande des Glases aufliegt, spielt eine Rolle. Wenn man den Rand nicht etwa durch Paraffinierung wasserabstoßend macht, kann die Abtropfgeschwindigkeit um ein Vielfaches vergrößert sein. Dies macht es auch verständlich, daß man nicht ohne weiteres reproduzierbare Verhältnisse erhält.

Dies zeigte sich auch bei der Prüfung der Frage, ob die abtropfende Wassermenge von der Länge des herabhängenden Streifens abhängt. Da nach PoISeuille bzw. nach (22) die Länge des Kapillarrohrs keine Rolle spielt, wäre eine gleichbleibende Tropfenzahl zu erwarten gewesen. Indem man nun denselben Löschpapierstreifen nacheinander einmal $12 \mathrm{~cm}$ und dann $6 \mathrm{~cm}$ etc. herabhängen ließ, wurden folgende Zeiten für die Bildung eines Tropfens gemessen

\begin{tabular}{rrrrrr} 
Länge & & \multicolumn{3}{c}{ Mittel } \\
$12 \mathrm{~cm}$ & 91 & 80 & 74 & 82 & $\mathrm{sec}$ \\
$6 \mathrm{~cm}$ & 103 & 91 & 86 & 93 & sec
\end{tabular}

Es ergaben sich also trotz der Regelmäßigkeit, mit der bei jeder Einstellung die Tropfen fielen, unregelmäßige Schwankungen der Meßwerte. Indessen sprechen die Zahlen doch dafür, daß die Strömungsgeschwindigkeit in langen und kurzen Streifen praktisch dieselbe ist. Vorausgesetzt ist dabei, daß die Steighöhe vom Niveau im Glas bis zum höchsten Punkt als verschwindend klein angenommen werden darf. Im anderen Fall ist zu berücksichtigen, daß nur der Höhenunterschied zwischen Wasserniveau und Streifenende den antreibenden 
hydrostatischen Druck liefert, daß aber andererseits der Reibungswiderstand auf der ganzen Strecke vom oberen eintauchenden Ende bis zum unteren zu überwinden ist. Bezeichnet man diese ganze Länge mit $L$ und die Steighöhe mit $l$, so ist die nach (22) sich ergebende Abflußmenge daher mit $\frac{L-2 l}{L}=1-\frac{2 l}{L}$ zu multiplizieren. Hängt der Streifen nur bis auf Niveauhöhe herab, d.h. ist $L=2 l$, so saugt sich zwar der Streifen mit Wasser voll, es findet aber kein Abtropfen mehr statt. Dies tritt indessen z.B. wieder ein, wenn man einen auch nur kurzen nassen Papierstreifen mittels Kapillarkraft anhängt. Ferner kann man beobachten, daß bei Schrägstellung des Papierstreifens die Tropfenbildung sich entsprechend verlangsamt, und hebt man das Ende auf die Höhe des Wasserniveau, so setzt die Funktion dieses Kapillarhebers ebenfalls ganz aus.

\section{Strömungslinien}

Bei der Behandlung des Fließvorgangs in porösen Körpern wurde stillschweigend vorausgesetzt, daß man es mit einer gerichteten Strömung zu tun habe. Inwieweit dies zutrifft, soll anhand einiger Fließbilder, die mit der einfachen Anordnung der Abb. 4 hergestellt wurden, gezeigt werden (Abb.7). Zu diesem Zwecke brachte man während des Einsaugens des Wassers an 4 bis 7 äquidistanten Punkten (Abstand 5 bis $9 \mathrm{~mm}$ ) mit Hilfe einer Schreibfeder Tuschetupfen auf. Dies in einigen $\mathrm{cm}$ Abstand von der Einsaugstelle, um ein Zerfließen der Tusche in der mit Wasser bedeckten Oberfläche zu vermeiden, auch nicht vor dem Versuch oder unmittelbar nach Durchgang der Fließfront. Diese ließ man erst einige $\mathrm{cm}$ weit vorrücken, damit das Wandern der Tusche dann nach Erreichung des stationären Fließzustandes vor sich gehen konnte. Wie aus Abb.7 ersichtlich, erhält man so ausgeprägte Stromlinienbilder, wie sie einer freien laminaren Flüssigkeitsströmung entsprechen. Während des Versuches kann man auch sehr schön beobachten, daß die Fließfront sich jeweils 上 zur Strömung einstellt. Ein Versuch dauert im allgemeinen weniger als 10 Minuten. Bild C ist mit Whatman 4, alle übrigen Aufnahmen sind mit Löschpapier hergestellt. Um nicht nur das Strömen in verschieden geformten Kanälen, sondern auch um Hindernisse herum zu zeigen, wurden in Streifen $F 2$ Einschnitte gemacht, und bei $G$ war das Papier auf einer Partie in der Größe eines Frankenstückes paraffiniert. 
Bemerkt sei, daß nicht jedes Färbemittel verwendbar ist, da diese gewöhnlich gemäß dem papierchromatischen Trennverfahren gänzlich mitgeführt werden, während schwarze Tusche deutliche Wegspuren hinterläßt. Man erkennt im übrigen, daß die so erzielten Strömungslinien sich mit Zunahme der Wegstrecke etwas verbreitern, indem sich

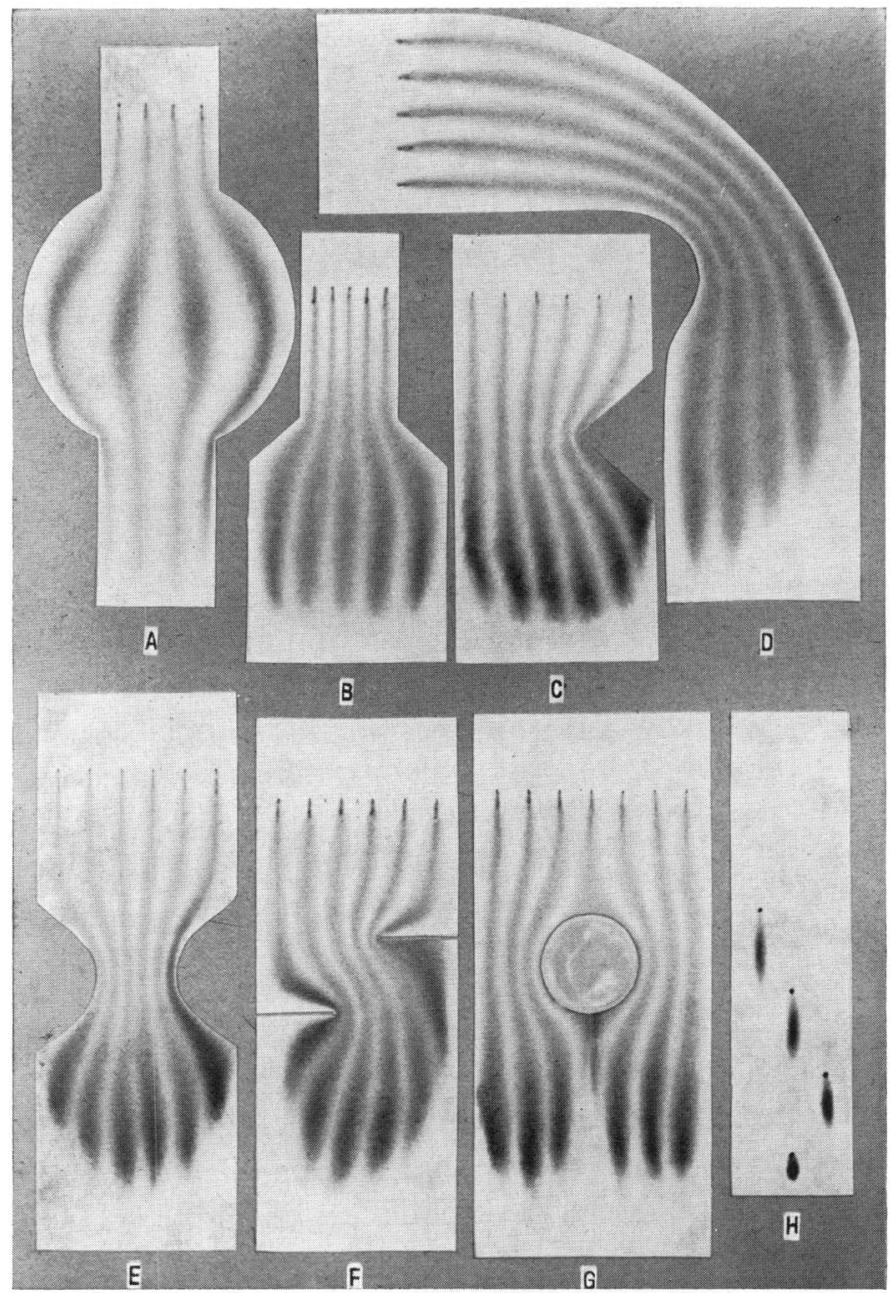

A bb. 7. Das Fließen in porösem Material, dargestellt durch Stromlinienbilder. ABCDE: Strömung in verschieden geformten Kanälen, F G: um Hindernisse, H: Nachströmen 
zu der gerichteten Strömung eine fortschreitende seitliche Diffusion zugesellt. Der Effekt ist indessen nicht so groß, daß man nicht brauchbare Stromlinienbilder von über $10 \mathrm{~cm}$ Länge erhalten kann. Das Verfahren dürfte daher auch für Demonstrationszwecke im Physikunterricht gute Dienste tun, besonders dann, wenn ein PoHLscher Stromlinienapparat nicht zur Verfügung steht. Es besitzt diesem gegenüber noch den Vorteil, daß die Bilder nach Herstellung leicht fixiert werden können. Man hat nur die nassen Proben mit der Rückseite auf ein Fließpapier zu legen und sie mit einem gewöhnlichen Papier leicht anzudrücken.

Physikalisches Institut der Universität Bern 